\title{
Philosophiques
}

\section{Guy Bourgeault, L'éthique et le droit face aux nouvelles technologies biomédicales, Montréal, P.U.M., 1990.}

\section{Lukas K. Sosoe}

Volume 21, numéro 1, printemps 1994

URI : https://id.erudit.org/iderudit/027269ar

DOI : https://doi.org/10.7202/027269ar

Aller au sommaire du numéro

Éditeur(s)

Société de philosophie du Québec

ISSN

0316-2923 (imprimé)

1492-1391 (numérique)

Découvrir la revue

Citer ce compte rendu

Sosoe, L. K. (1994). Compte rendu de [Guy Bourgeault, L'éthique et le droit face

aux nouvelles technologies biomédicales, Montréal, P.U.M., 1990.]

Philosophiques, 21(1), 282-289. https://doi.org/10.7202/027269ar d'utilisation que vous pouvez consulter en ligne.

https://apropos.erudit.org/fr/usagers/politique-dutilisation/ 


\title{
Guy Bourgeault, L'éthique et le droit face aux nouvelles technologies biomédicales, Montréal, P.U.M., 1990.
}

\section{par Lukas K. Sosoe}

L'ouvrage de Guy Bourgeault s'intitule : prolégomènes pour une bioéthique. Titre éloquent et ceci à deux titres au moins : d'abord, tout indique qu'il s'agit bien d'un programme; ensuite, d'une conception particulière de la bioéthique puisque l'auteur prend soin d'écrire, nous ne pensons pas le trahir ici, « pour une bioéthique ». Modestie ou stratégie d'argumentation, il est difficile de dire avec exactitude. Cependant une chose est certaine; la définition qui est donnée de la bioéthique s'éloigne quelque peu du sens commun de ce terme pour désigner une approche, une méthode et, une fois encore au risque de nous répéter : un projet. Il s'agit donc d'une approche nouvelle, orientée vers la prise de décision, des enjeux éthiques liès à l'utilisation croissante des technologies touchant directement à la vie humaine et à la santé (p. 28). Plus loin l'auteur renchérit : la bioéthique est un « champ d'études, l... ayant pour objet des pratiques et des processus, (définis par le développement des technologies de la vie et de la santê) et une approche pour la décision et l'action, plutôt qu'une discipline » (p. 2g). Elle se distingue non seulement d'une déontologie professionnelle pour les médecins. Sa méthode est casuistique et

\begin{abstract}
[...] résolument orientée vers la prise de décision dans une société séculière et pluraliste. Approche donc nécessairement pluridisciplinaire et systémique [...] un acte démocratique, dans la mesure où la discussion des enjeux fondamentaux s'impose à la bioethique comme un devoir et comme une tâche démocratique essentielle d'information et de formation. Puisque c'est de la vie de tous et de toutes qu'il s'agit, individuellement et collectivement $\gg$ (p. $3^{\mathrm{I}}$ ).
\end{abstract}

La question qui vient tout d'abord à l'esprit est de savoir comment réussir, plus modestement écrire, des prolégomènes à une approche aussi vaste, une entreprise aussi colossale qui engage non seulement la responsabilité des experts de toutes les disciplines, mais encore celle, beaucoup plus fondamentale, des citoyens que nous sommes. Comment pourrait bien se présenter, non pas la réalisation, mais une approche aussi générale, engageant donc l'ensemble du savoir, plus précisement du savoir humain puisque, selon la thèse de l'auteur : « les questions fondamentales de la bioéthique débordent largement celle-ci et sont communes aux efforts de réflexion portant sur les dimensions éthiques et juridiques du développement des technologies en général, en vue d'un contrôle social de ce développement » (p. 32) ?

C'est pour mieux répondre à cette question que nous aimerions, après une brève esquisse du plan de l'ouvrage, suivre ici les grandes étapes de l'argumentation de Bourgeault en insistant particulièrement sur certaines thèses et prises de position plutôt que sur d'autres.

Le livre est divisé en trois grandes parties qui, loin d'être uniquement un alignement de différents sujets, forment une unité autour de laquelle s'articulent les grandes idées.

La première partie : l'éthique et le droit face à la technologie. Plaidoyer pour un nouvel esprit éthique, établit un diagnostic négatif de la situation du droit et de 
l'éthique face aux nouvelles technologies. Négatives en ce sens que le droit et l'éthique doivent désormais tenir compte des nouveaux pouvoirs liés au développement technologique. Cette partie fait également état de l'apparition de nouveaux problèmes auxquels ne sauraient plus faire face les anciennes morales, c'est-à-dire les morales traditionnelles, « ébranlées dans leurs fondements mêmes » et désormais insuffisantes et peu aptes pour « la conduite des groupes et des collectivités » (p. 44), d'autant plus qu'elles osent «enracinées dans un passé et valables pour des solidarités plus limitées » (p. 51). Bref pour Bourgeault, nous assistons d'une par, à une mutation sociale et culturelle qui

[...] entraîne l'inadéquation des règles et des normes élaborées dans un cadre général en voie d'effacement, sinon déjá totalement disparu. D’autre part et surtout làl l'avènement de la technologie et [del ses dêveloppements [qui] situent les individus et les collectivités dans de vastes réseaux d'interdépendances structurelles diverses, à l'échelle de la planète, en même temps qu'elles conditionnent leur futur, avec celui de la planète [...] (p. 50).

D’où la nécessité et le sens de nouvelles interrogations dont la bioéthique est désormais porteuse. Celle-ci doit désormais assumer la réconciliation devenue inévitable entre l'éthique, le droit et la technologie (p. $78 \mathrm{sq}$.).

La deuxième partie, Autour de la vie, discute les enjeux et repères éthiques et juridiques. Elle constitue une véritable leçon d'anthropologie culturelle sur les diverses conceptions de la mort et, partant, de la vie, qui ont jalonné non seulement les civilisations occidentales, mais aussi l'Égypte et les sociétés primitives. Cette leçon est destinée à nous préparer à saisir pourquoi, dans un monde sécularisé qui est celui de la démocratie, caractérisée par le pluralisme, la vie est désormais « livrée à notre responsabilité. » Il y a plus. La deuxième partie s'achève sur un thème qui peut être choquant pour les uns, appréciable pour les autres, mais dans tous les cas, très peu, et qui mérite d'être remarqué et sérieusement discuté: De la vie à la personne : vers un nouveau paradigme.

La troisième partie, responsabilité partagée, se compose de deux chapitres : I) Responsabilité professionnelle : quelques repères pour une éthique du droit de l'intervention professionnelle dans le champs biomédical; et 2) Responsabilité sociale : le contrôle social du développement et de lutilisation des technologies de la vie et de la santé. Assez longs, ces titres ont l'avantage d'être explicites et ne demandent pas de commentaires supplémentaires. Disons, en un mot, que l'auteur y développe les bases de la bioéthique, de l'éthique nouvelle, bases qu'il appelle contractuelles, placées sous le signe du contrat et de la convivialité. Il précise : « Le contrat dit l'égalité des parties. La convivialité exprime leur étroite association dans une action commune et, si je puis dire, dans un sort fondamentalement commun, celui d'une commune humanité dont la responsabilité doit désormais être assumée de façon solidaire » (p. 197). Bourgeault explicite les composantes de cette conception contractualiste et en expose trois concepts fondamentaux: compétence, autonomie personnelle et responsabilité (p. 199-204).

Programme colossal, on l'aura remarqué, et d'une certaine manière aussi bien admirable que provocateur. En bien des points, il ne manque pas d'audace. Nous le montrerons. Plutôt que de nous attarder sur son ampleur, sur les défis que l'auteur se lance et que, du moins nous le souhaitons, il pourra un jour sortir de la condition de simples prolégomènes, nous voudrions ici, la critique ne devant pas exclure l'admiration, revenir sur certains thèmes centraux. Il 
faut toutefois faire remarquer que les prétentions de la critique ici developpée sont plus modestes, plus modestes en tout cas que celles que revendique l'auteur qui de toute évidence « veut simplement, comme philosophe, apporter son concours à un effort collectif et pluridisciplinaire l...J de définition de la bioéthique et de son objet » (p. 19).

Malgré les efforts de l'auteur pour distinguer l'éthique du droit, exercice scolaire, mais qui, somme toute, a son importance, la thèse de la perte de repères mérite d'être discutée. D'un côté, Bourgeault argumente que les morales anciennes sont dépassées par les nouvelles technologies. D'un autre côté, il nous propose tout de même tout un catalogue de principes qui sont censés initier ce qu'il appelle le nouvel esprit éthique. On aimerait savoir d'où le nouvel esprit éthique tire ses principes d'autonomie, de compétence et de responsabilité ? Ou alors ces principes sont tout à fait nouveaux, ou alors la thèse mérite d'être quelque peu modifiée. Il serait aberrant de dire que les principes mentionnés ci-dessus sont une invention de la bioéthique et n'ont rien à voir avec la tradition bi-millènaire de l'éthique. Comment rendre l'appel à un nouvel esprit éthique compatible avec des termes de l'éthique philosophique tels que l'autonomie et la responsabilité, puisque de toutes les façons le troisième principe, la compétence, serait aussi bien évident pour un Grec que pour un Romain, sans parler de notre condition contemporaine? Certes l'auteur donne des raisons et se donne la peine de qualifier la thèse. L'expérience du Québec lui sert d'exemple, un Québec où « l'ordre économique, social et politique [...] fut perçu jusqu'à recemment comme appartenant à un ordre naturel immuable, connu par ceux qui détiennent le pouvoir et font la loi ». Aussi y a t-on présentè le régime de la chretienté comme un ordre naturel, une loi naturelle dictant l'obéissance et la soumission à l'autorité. Seulement, à voir les choses de près, l'auteur n'a pas tenu compte de sa distinction entre éthiques et morales traditionelles. Comment comprendre les principes mentionnes plus haut comme des principes d'un nouvel esprit éthique? En quoi est-ce que la responsabilité et l'autonomie, pour ne citer que ces deux, sont fondateurs d'un nouvel esprit éthique? Qu'on conçoive différemment et selon les époques la responsabilité, voilà une chose; mais que l'on en fasse des principes d'un nouvel esprit éthique, voilà qui dépasse tout entendement, pour peu que l'on tienne compte de l'histoire des théories éthiques. I'auteur est mieux placé pour le savoir que la plupart des bioéthiciens, surtout nord-américains, auxquels manque souvent la profondeur historique. Il y a donc visiblement, malgré la distinction que nous acceptons, un glissement de sens de l'éthique vers la morale, les mœurs particulières, et une redoutable réduction des théories éthiques aux mœurs chrétiennes et peut-être même une réduction de la morale chrétienne à ce que nous appellerions une morale matérialisée dans un contexte socio-culturel précis.

Il appert donc que s'il doit y avoir un appel à un nouvel esprit éthique, celui-ci doit montrer en quoi il se veut tout à fait nouveau, non seulement en vertu du développement technologique et des problèmes inédits que posent celui-ci, mais encore en vertu du changement radical des principes. Bourgeault ne l'a pas pas fait. Même si des prolégomènes ne peuvent dire tout ce dont ils sont concrètement les prolégomènes, la thèse est trop importante pour la compréhension du gigantesque projet de l'auteur pour qu'elle ne soit pas prise à la 
légère. L'essentiel s'y joue, dans la mesure où elle légitime la nouvelle aventure d'un nouvel esprit éthique.

La vie livrée à notre responsabilité, thèse audacieuse, il faut le reconnaître, puisque malgré notre condition d'héritiers de la modernité, malgré le discours du manque de repères, force est de constater l'appel à des valeurs comme si celles-ci n'ont pas été touchées par le phénomène dont nous parle l'auteur. Que de gens pensent encore aux valeurs comme si elles étaient encore dans le ciel de Platon! Que de gens (non bello sed pace Guy Durand) pensent encore à des « grilles de valeurs » comme si ces valeurs existaient encore et surtout existaient indépendamment de procédures de décisions que des agents autonomes et égaux en droit, jouisssant de la même dignité, auraient adoptées dans une situation de dialogue idéale! Toutefois, comme le lecteur pourrait l'avoir remarqué, c'est ici que Bourgeault ne prend pas toute la mesure de sa propre thèse qui, de toute évidence, est fondamentalement anti-métaphysique! Pour une fois l'homme devient la mesure de toute chosee en matière de vie et de mort, malgré les incertitudes qui ne sont que les conséquences de notre condition sociale et politique moderne (et même postmoderne) pluraliste. L'auteur offre à l'appréciation de ses lecteurs le principe de la présomption en faveur de la vie : « la vie humaine, livrée à notre responsabilité, doit avoir notre faveur, nous nous devons à nous-même comme aux autres dont nous sommes solidaires de favoriser sa préservation et sa croissance. Faute de quoi, fragile en tous ces moments, elle n'a pratiquement aucune chance d'avenir ». Mais là également nous restons sur notre faim. Guy Bourgeault nous parle de prolégomènes. On est en droit de s'attendre aux fondements mêmes de la bioéthique. Certes, il le reconnaît lui-même en toute lucidité : «plus d'un lecteur, sans doute estimera être resté sur son appétit ». Mais il se défend : « l'objectif était I...I d'aider à problématiser l... en problématisant » (p. 237). Toutefois cela ne suffit pas au lecteur affamé, car livrer la vie à la responsabilité humaine est une provocation si on considère toutes les thèses que développent les bioéthiciens qui sont surtout de tendance chrétienne avouée ou inavouée. Il aurait donc fallu argumenter et non pas seulement affirmer. Il aurait fallu montrer en quoi nous sommes devenus, d'une certaine manière, les dépositaires de la vie que nous pouvons changer, manipuler, améliorer, modifier. Redoutable prise en charge de la vie face à beaucoup de théories bioéthiques qui, sans se l'avouer, demeurent dans le sillon de la pastorale, même si elles trouvent les directives du Vatican trop restrictives. Bourgeault nous laisse sur notre faim. Nous aurions bien aimé savoir pourquoi nous devenons dépositaires de la vie et pourquoi, ici encore, les théories éthiques traditionnelles, pour autant qu'elles soient modernes et pas confondues avec des « morales locales » et des théories éthiques par trop métaphysiques, tomberaient en désuétude face à notre programme de la prise en charge de la vie. Prise en charge de la vie, un projet éminemment moderne qui ne fait plus référence à des valeurs métaphysiques, mais conçoit la dignité humaine en termes séculiers. Ce qu'accomplissait la Commission canadienne de réforme du droit démontre, au plan philosophique, comment, sous la poussée d'éléments modernes du droit, les catégories pré-modernes disparaissent. Ce processus ne signifie pas une absence de valeurs ou absence de repères, mais la traduction, en termes modernes, de la dignité humaine fondée, tour à tour, sur un ordre cosmique, sur la filiation 
divine de l'homme, etc. Or cette traduction est nécessaire au pluralisme que nous vivons, pluralisme qui est en lui-même une valeur, puisqu'il repose sur la tolérance et sur la valeur de l'homme en tant qu'homme. Et c'est bien là l'horizon axiologique de notre culture démocratique.

C'est ici qu'intervient le problème de l'anthropologie. Bourgeault soutient qu'un renvoi implicite ou explicite à une anthropologie antérieure n'est plus possible puisque l'homme devient ainsi son propre créateur. Il nous faut donc une éthique et un droit sans anthropologie (p. 88). Cette thèse ne semble pas évidente. $\mathrm{Si}$ « anthropologie antérieure » se réfère ici à une série de théories où une définition essentialiste est donnée de l'être humain, il est difficile de ne pas suivre l'auteur. Mais la thèse nous paraîtrait quelque peu triviale. On pourrait lui attribuer un sens plus fort, selon lequel l'éthique comme le droit n'auraient plus besoin d'anthropologie, du moins dans le contexte du nouvel esprit éthique. Seulement, prise dans ce sens fort, la thèse s'opposerait quelque peu à une partie de l'entreprise de l'auteur; car indépendamment du fait qu'il est impensable de concevoir une éthique dans le sens large du terme sans anthropologie, l'auteur lui-même nous présente diverses conceptions de la vie et de la mort, présentation dont la valeur, à notre avis, dépasse la simple érudition, dans la mesure où elle est justement censée nous indiquer la difficulté qu'il y a de nos jours à trouver des repères normatifs. Il voulait nous montrer, en d'autres termes, que :

[...] les débats à la fois éthiques et politiques des dernières années autour de la vie et de la mort ont porté la marque, souvent implicite, de la pluralité des visions et des opinions touchant la vie et sa qualité. D'où l'opposition courante, en vue d'une conciliation souhaitée, sinon réussie - et peut-être impossible -, entre les exigences du respect intégral ou absolu de la vie humaine et de son caractère sacré, et celles de la prise en compte de sa qualité dans les décisions touchant l'opportunité d'interventions médicales (p. I5I).

Si tel est donc le cas, il y a bel et bien anthropologie, aussi minimale soit-elle. La question n'est pas une éthique et un droit sans anthropologie, mais plutôt quelle (subtile) anthropologie nous faut-il pour une fondation de l'éthique et du droit en démocratie ? Ne nous restera donc à repenser le programme dont Günther Patzig s'est fait le défenseur il y a encore quelques années : «Ethik ohne Metaphysisk »! Là encore, au risque de nous répéter, nous nous situons de plain-pied dans une perspective moderne. On comprend donc, dans ce contexte, pourquoi Rawls, par exemple, insiste sur le fait que sa théorie de la justice est politique et non métaphysique. Si anthropologie signifie nécessairement une vision de l'homme qui fait référence à des éléments qui transcendent l'humanité, on suivra Bourgeault sans peine. Mais si « éthique et droit sans anthropologie » veut dire qu'il faut en finir avec toute anthropologie, même celle qu'exige notre condition de la modernité, alors on voit mal comment se peut déployer un discours éthique, même dans le cadre du nouvel esprit éthique. On voit mal également quel sens il faut accorder à toute la deuxième partie de son ouvre. Surtout on comprendra mal Bourgeault quand il écrit lui-même: « Dans un cas, donc, la bioéthique exige que l'éthique s'ouvre à l'anthropologie, à la psychologie, à la sociologie, et bien sûr à la métaphysique (sic). Dans l'autre, qu'elle s'ouvre à l'économie, à l'administration, à la comptabilité, à la politique, à l'information, à l'éducation » (p. 238-239, nos 
italiques). Passage qui prouve que le projet du nouvel esprit éthique ne peut se passer d'anthropologie.

Ethique et droit sans anthropologie, éthique et droit parce que nous n'avons plus de repères normatifs ! Et pourtant Bourgeault réfute la neutralité axiologique qu'avancent certains scientifiques. Il remarque fort justement que les scientifiques « ont revendiqué pour la science, avec une insistance croissante depuis bientôt deux siècles, une neutralité axiologique et éthique généralement non critiquée et qui paraît devoir consommer le divorce signalé plus haut » (p. 68). Il attire également l'attention sur les conditions de production du savoir scientifique et sur les représentations idéologiques (p. 6g) qui sont, sinon aptes à réfuter, du moins sont à même de minimiser grandement la thèse de la neutralité. Deux problèmes se posent :

I) la science est bel et bien porteuse des représentations qui ne sont pas neutres. Mais cela signifie-t-il déjà qu'on ne peut distinguer le savoir théorique scientifique (raison théorique) d'un savoir normatif (raison pratique) ? Lesdites représentations idéologiques qui sous-tendent les sciences et la technique sont-elles de même nature que nos discours évaluatifs? Le principe de Poincaré (connu sous le problème de Hume), selon lequel de l'être nous ne pouvons déduire le devoir-être, est-il véritablement mis en échec (p. 8o) ? Qu'un scientifique évalue en citoyen ce qu'il fait et qu'il prenne même position, cela signifie-t-il que son activité en tant que scientifique s'identifie à son évaluation en tant que citoyen?

2) à supposer que la revendication de la neutralité axiologique soit une erreur de la part des scientifiques, Bourgeault aurait donc tout à fait raison. Seulement, ce ne serait pas dans son intérêt, car il voudra précisément dire qu'il existe encore de nos jours des représentations normatives, donc bel et bien des repères.

Un dernier aspect important du projet de Bourgeault nous inquiète plus particulièrement. C'est l'ambition de totalisation du savoir et de sa mise à la disposition du politique, projet intimement lié, on se souviendra, au développement même de la philosophie, surtout en Allemagne il y a à peine deux ans. Loin de nous l'idée de penser que le savoir doit rester localisé dans des lieux quasiment sacrés, universités ou autres. On les appelera comme on voudra. Loin de nous également l'idée que la vie sociale et politique démocratique doit être rigoureusement séparée de la production du savoir. Mais on peut bien se poser tout de même la question de savoir si ce n'est trop demander aux bioéthiciens que d'exiger d'eux de devenir les nouveaux encyclopédistes. Bourgeault ne transforme-t-il pas en vertu la nécessité dans laquelle se voit la bioéthique de se donner une définition plus précise? Il y a certes des écrits qui vont dans ce sens. Pensons tout d'abord à ce qui a motivé l'éthique de la discussion d' Apel et de Habermas. Pensons également aux Strategien der Humanität. Zur Ethik öffentlicher Entscheidungsprozesse de O. Höffe. Toutefois il s'agit moins de totaliser le savoir que de réfléchir, dans les conditions qui sont celles des sociétés modernes complexes et de leurs valeurs, sur les processus légitimes de prises de décisions politiques, processus de prises de décisions qui peuvent, bien évidemment, bénéficier de l'apport de quelques experts. On peut se demander si la tâche qu'assigne Bourgeault à la bioéthique ne dépasse pas de beaucoup celle-ci. Car, à le suivre, on a l'impression que la bioéthique 
deviendrait, dans le monde contemporain une équivalente à la reine des sciences, pour utiliser une expression qui désignait, au Moyen-Âge, la théologie. Disons même qu'elle deviendrait une sorte de théologie séculière au service de laquelle les autres se mettraient. L'arène publique deviendra certes un lieu de débats publics. Mais elle se transformerait elle aussi en une sorte d'Église séculière où les bioéthiciens joueraient le rôle de clergé. Celui-ci décidera de la latitude de droits et libertés, puisque la bioéthique se veut en plus « orientée vers la prise de décisions » cas par cas. On pourrait bien se demander pourquoi la bioéthique, après cette prodigieuse synthèse, adopterait nécessairement la casuistique comme méthode. Pourquoi est-ce que la nature des problèmes à résoudre n'autoriserait pas une solution globale?

Ici également, au plan méthodologique, on a l'impression que la bioéthique doit nécessairement se distinguer de toute autre théorie éthique. Comment peut-on faire de la casuistique en l'absence de grands principes tels que justement l'autonomie, la responsabilité et autres? Si on se limitait à la casuistique, comment procéder donc à la justification de ces principes, c'est-à-dire comment montrer qu'ils ne sont pas arbitraires?

Cette interprétation ne semble pas faire droit à l'auteur qui n'oserait jamais penser une telle chose. Du moins, c'est à supposer ! Toutefois, à le suivre encore, il s'accomplirait, dans la bioéthique, non seulement la synthèse des échanges entre experts de diverses disciplines, mais encore une discussion « des enjeux fondamentaux [...] comme devoir et comme une tâche démocratique essentielle d'information et de formation $\gg$ (p. 3i). Comment comprendre donc autrement cette mission de la bioéthique surtout si l'on tient compte du fait, a) qu'elle s'approprie l'étiquette d'acte démocratique et b) qu'elle ne définit aucune condition d'un dialogue démocratique idéal. Le chapitre sur les repères (p. 199-204) ne nous en dit rien. Il développe au contraire des principes de base de la bioéthique.

Par où l'on comprend que la bioéthique prend la place des projets de société qui existaient il y a quelques temps encore. La thèse de l'absence de repère entreprend l'érosion des valeurs modernes dont on reconnaît bien entendu le pluralisme tout en en niant l'existence. Or reconnaître le pluralisme, c'est de toute évidence affirmer qu'il existe des valeurs, mais qu'elles sont compétitives ou concurrentes. Vouloir dire le contraire n'aboutit pas seulement à l'incohérence; c'est en plus méconnaître l'horizon du monde vécu. Le projet bourgaltien me semble comporter un autre danger : précisément celui de l'encombrement du monde vécu par une expertise accrue. La prise en compte du monde vécu nous fait découvrir non seulement la vision de chaque pratique, chaque profession, mais encore l'idée de la bonne pratique de telle ou telle profession. Telle qu'elle est définie, la bioéthique peut tout normer. Justement parce qu'il n'y a plus de repères, il revient à la bioéthique de proposer des balises. Nous parlons de danger, donc de risque. Il ne s'agit pas pour nous de condamner les principes que propose Bourgeault. Au contraire! Ce que nous exigerions, c'est qu'il explique comment nous parvenons à les établir en tant que philosophe si nous ne prenons pas en compte l'horizon de notre culture démocratique, l'horizon de notre Lebenswelt démocratique, comme point de départ. Vouloir réaliser l'approche bioêthique que nous propose Bourgeault, c'est confier, au-delà des experts des différentes disciplines, le discours 
pratique à un certain nombre de gens qui se nomment bioéthiciens et qui décideront, peut-être au nom de principes louables, de choses qui nous regardent. La source des valeurs, c'est bien eux !

Malgré toutes ces critiques le livre de Bourgeault reste le meilleur livre en bioéthique dans le monde francophone qui ose poser de grands problèmes. L'horizon du livre est immense et ses intuitions profondes. On y trouve d'immense références aussi bien à la littérature anglo-américaine que continentale. En cela l'ouvrage est un exemple à suivre. On peut regretter toutefois l'absence de la discussion allemande. Puisqu'il ne s'agit que d'une modeste contribution aux fondements de la bioéthique, puisqu'il ne s'agit que de prolégomènes, attendons la suite. On sera peut-être plus éclairé. Pour lors, répétons-le tout de même encore une fois, nous restons sur notre faim.

Département de philosophie

Université de Montréal 